\title{
The Influence of Amino Acids on Copper Uptake by Rat Liver Slices*
}

\author{
Daisy I. M. Harris $\dagger$ and Andrew Sass-Kortsak + \\ (From the Department of Paediatrics, Faculty of Medicine, University of Toronto, and The \\ Research Institute, The Hospital for Sick Children, Toronto, Ontario, Canada)
}

\begin{abstract}
Summary. Addition of physiological concentrations of 20 L-amino acids to the incubation medium facilitates the uptake of copper by liver slices. This effect can be observed in a wide range of initial concentrations of copper in the medium, including the low physiological concentrations. The uptake of copper was not influenced by the addition of sodium lactate or D-glucose to the incubation media. The facilitation of copper uptake by amino acids is abolished under anaerobic conditions. It is not affected by the presence of 2,4-dinitrophenol. The type of transport and its exact mechanism have not been established, but this type of transport does not fulfill the criteria for classification as simple diffusion.
\end{abstract}

\section{Introduction}

In a preceding paper, it was shown that, in addition to copper in ceruloplasmin and that bound to albumin, there is a third small fraction of copper in human serum that is bound to amino acids. It was suggested that this latter fraction may be involved in the transport of copper between the blood and the tissues (1).

The experiments to be described in this paper were designed to test the validity of the latter hypothesis by the use of an in vitro system. The model of surviving liver slices was used for this purpose since it is known that in the intact human, rat, and other animals, a large percentage of copper that enters the bloodstream is taken up by the liver $(2,3)$.

\footnotetext{
* Submitted for publication May 31, 1966; accepted December 30, 1966.

Supported by funds from the John A. Hartford Foundation, New York, N. Y.

Presented in part at the annual meeting of The Canadian Federation of Biological Societies, Halifax, Nova Scotia, June 1964.

† Present address: Zifkin Biological Laboratories, Toronto, Ontario, Canada.

$\ddagger$ Address requests for reprints to Dr. Andrew SassKortsak, 555 University Avenue, Toronto, Ontario, Canada.
}

\section{Methods}

We used the precautions specified in the preceding paper to avoid contamination with copper (1).

The basic incubation medium was Krebs-Ringer bicarbonate (KRB) buffer (4), which was pregassed with a mixture of $95 \%$ oxygen and $5 \%$ carbon dioxide (unless otherwise specified). To keep copper in solution, in most instances we added $1 / 10 \mathrm{vol} / \mathrm{vol}$ of dialyzed plasma or serum to the KRB buffer. We obtained this serum or plasma from rats and dialyzed it for 48 hours at $4^{\circ} \mathrm{C}$ against several changes of large volumes of KRB buffer to remove small molecules, including amino acids. We took extreme care to avoid contamination with copper during this procedure. The $\mathrm{pH}$ of the dialyzed serum or plasma was adjusted to 7.4 with $0.1 \mathrm{~N}$ sodium hydroxide and $0.1 \mathrm{~N}$ hydrochloric acid before its addition to the KRB buffer.

In some experiments, human serum albumin was added instead of the dialyzed rat serum or plasma. The albumin preparation was lyophilized Cohn Fraction V.1 The albumin-containing media were prepared by dissolving the appropriate quantity of albumin in KRB buffer. A final step of purification was extensive dialysis of these solutions as specified for serum above. The $\mathrm{pH}$ of each

1 This was prepared by the Connaught Medical Research Laboratories in Toronto from outdated human plasma collected by the Canadian Red Cross Society from volunteer donors. The preparation was kindly donated to us through the courtesy of Dr. A. M. Fisher. A single batch of this preparation was used for all experiments; its copper content was determined by the method of Eden and Green (5). 
TABLE I

Mixture of amino acids added

\begin{tabular}{lc}
\hline \multicolumn{1}{c}{ Amino acid } & Concentration* \\
\hline & $m g / 100 \mathrm{ml}$ \\
L-Asparagine & 0.58 \\
L-Aspartic acid & 0.03 \\
L-Citrulline & 0.50 \\
L-Cysteine· $\mathrm{HCl}$ & 0.86 \\
L-Glutamic acid & 0.70 \\
L-Glutamine & 8.30 \\
Glycine & 2.27 \\
L-Histidine & 0.97 \\
L-Isoleucine & 1.32 \\
L-Leucine & 2.66 \\
L-Lysine $\cdot \mathrm{HCl}$ & 7.25 \\
L-Ornithine· $\mathrm{HCl}$ & 0.92 \\
L-Phenylalanine & 1.37 \\
L-Proline & 4.30 \\
L-Serine & 1.12 \\
L-Threonine & 4.40 \\
L-Tryptophan & 1.68 \\
L-Tyrosine & 2.23 \\
L-Valine & 2.67 \\
L-Arginine $\cdot \mathrm{HCl}$ & 3.88 \\
&
\end{tabular}

* Some L-amino acids were added to the media in the same concentrations as they occur in plasma of normal rats (6). The remainder were added at their respective concentrations in normal human plasma (7).

solution was adjusted to 7.4 before its use but after the addition of amino acids, if applicable.

L-Stereoisomers of twenty amino acids were added to the incubation medium, when indicated, in quantities necessary to achieve final concentrations in the physiological range $(6,7)$ as shown in Table $I$.

Copper was added in the form of cupric chloride or acetate, dissolved in a small volume of water. A solution of $\mathrm{Cl}_{2}{ }^{-{ }^{-4}} \mathrm{Cu}$ of very high specific activity $(10$ to $20 \mathrm{mc}$ per $<1.0 \mu \mathrm{g}$ of copper) served as the label (8). Unless stated otherwise, copper was added in a $1.6 \times 10^{-8} \mathrm{M}$ concentration, equivalent to $10 \mu \mathrm{g}$ per $100 \mathrm{ml}$, which is within the range of physiological concentrations of nonceruloplasmin-bound copper in human serum (9). Most of the copper contained in the dialyzed rat serum that was added to the medium was in ceruloplasmin and, therefore, unexchangeable. The albumin-bound copper in rat serum is only $1 \mu \mathrm{g}$ per $100 \mathrm{ml}(10)$ and, therefore, negligible at the dilution used. Further additions to the media were made as specified in the descriptions of the particular experiments.

Slices were prepared from the livers of male rats of the Wistar strain, 300 to $400 \mathrm{~g}$ in weight. Their diet had been Master's laboratory cubes ${ }^{2}$ before they were fasted for approximately 24 hours prior to the experiment. The animals were killed by quick exsanguination while they were under light ether anesthesia. The liver was removed from each animal and dropped immediately into cold oxygenated KRB buffer. Cubes were cut from the two larger lobes of each liver, and these were kept in ice-

2 Maple Leaf Mills, Toronto, Ontario, Canada. cold KRB buffer that was continuously gassed with $95 \%$ oxygen and $5 \%$ carbon dioxide until used (11).

Slices were prepared from the cubes as quickly as possible with a Stadie-Riggs microtome and blade (12). A single slice (weighing between 50 and $120 \mathrm{mg}$ ) was then dropped into $3.0 \mathrm{ml}$ of medium contained in a $20-\mathrm{ml}$ beaker. The beakers containing the media were kept on ice. As soon as one cube had been sliced, the beakers with the slices were placed in a Dubnoff metabolic incubator.

Incubation was carried out at 37 to $38^{\circ} \mathrm{C}$ under an atmosphere of $95 \%$ oxygen and $5 \%$ carbon dioxide unless otherwise specified. We used commercially available gas mixture ${ }^{3}$ that was bubbled through water before being passed through the incubator at a rate of 8 to $9 \mathrm{~L}$ per minute. The samples were shaken constantly at the rate of 90 oscillations per minute.

After incubation, the slice was removed from its medium with a fine pair of forceps, quickly dipped twice in $\mathrm{KRB}$ buffer, blotted, weighed, and placed in the bottom of a counting tube.

The gamma emission of ${ }^{84} \mathrm{Cu}$ contained by the slice was assayed by a well-type scintillation counter. Samples of each medium in which slices had not been incubated were also counted. Counts were corrected for background and for decay based on 12.8 hours half-life of ${ }^{84} \mathrm{Cu}$. A further correction was made by subtracting the average activity taken up by slices that had only been quickly dipped in the media, but had not been incubated, from the activity contained in each incubated slice. This latter correction never exceeded $2 \%$ of the total activity and was usually less.

Each result was expressed as the ratio between the activity in a unit weight of the slice and the initial activity in a unit weight of the medium. A correction for the slight difference between the specific gravity of the medium and of the slice was not made. In some instances, we calculated the uptake of copper by the slices, having made the assumption that the uptake of ${ }^{84} \mathrm{Cu}$ paralleled that of copper.

The arithmetic mean, the standard deviation, and the standard error of the mean were calculated for each group of experiments. We used Student's $t$ test $(13,14)$ to assess the significance of the differences between the means.

\section{Results}

In the first series of experiments, liver slices were incubated in media that contained $1.6 \times 10^{-6}$ $M$ concentration of copper with and without physiological concentrations of $20 \mathrm{~L}$-amino acids (Table I). All media contained $\frac{1}{10}$ vol of dialyzed rat serum.

The results are shown in Figure 1. It is apparent that the uptake of ${ }^{64} \mathrm{Cu}$ by the slices incu-

3 Ohio Chemical Canada, Ltd., Toronto, Ontario. 
TABLE II

Effect of $\left[\mathrm{Cu}^{++}\right]$in the medium on the uptake of ${ }^{64} \mathrm{Cu}$ by liver slices

\begin{tabular}{cllcc}
\hline \hline \multirow{2}{*}{$\begin{array}{c}\text { Time of } \\
\text { incubation }\end{array}$} & \multicolumn{1}{c}{ Type of medium } & \multicolumn{3}{c}{ Initial $\left[\mathrm{Cu}^{++}\right]$in medium $\times 10^{-6}$ mole per $\mathrm{L}$} \\
\cline { 4 - 5 } $\min$ & & 1.6 & 5.0 & 50.0 \\
15 & Control & & & \\
& Amino acids added & $0.25 \pm 0.02(7)^{*}$ & $0.23 \pm 0.04(7)$ & $0.50 \pm 0.06(6)$ \\
\multirow{2}{*}{30} & Control & $0.06 \pm 0.08(8)$ & $0.40 \pm 0.05(6)$ & $1.09 \pm 0.08(7)$ \\
& Amino acids added & $0.89 \pm 0.05(7)$ & $0.61 \pm 0.05(8)$ & $2.05 \pm 0.37(8)$ \\
\multirow{2}{*}{60} & Control & $0.61 \pm 0.03(8)$ & $0.85 \pm 0.09(7)$ & $2.04 \pm 0.17(8)$ \\
& Amino acids added & $1.28 \pm 0.13(8)$ & $1.40 \pm 0.18(8)$ & $3.55 \pm 0.55(8)$ \\
& Control & $1.12 \pm 0.08(8)$ & $1.03 \pm 0.05(8)$ & $2.84 \pm 0.53(7)$ \\
& Amino acids added & $1.81 \pm 0.24(8)$ & $1.50 \pm 0.16(7)$ & $4.20 \pm 0.62(7)$
\end{tabular}

* Each result shown for ${ }^{64} \mathrm{Cu}$ uptake is the average of ratios of activity per milligram of slice/activity per milligram of medium \pm standard error of the mean. Figures in parentheses represent the numbers of samples included for the means.

bated in media to which amino acids had been added was faster than the uptake by the slices incubated without addition of amino acids. These differences at 15,30 , and 60 minutes are significant at the $0.1 \%$ level $(p<0.001)$. The difference between the means at 120 minutes is not significant $(p>0.05)$. We suspect that, in the incubations without added amino acids, there is a gradual release of amino acids into the media with time that may eventually abolish the difference in the rates of uptake. Alternatively, it may be that a steady state is about to be reached in these experiments by 120 minutes and that this steady state is the same both in the presence and absence of added amino acids.

The effect of the concentration of copper in the medium on the uptake of copper into the slices

\begin{tabular}{|c|c|c|c|}
\hline \multirow{2}{*}{$\begin{array}{l}\text { Copper } \\
\text { concen- } \\
\text { tration }\end{array}$} & \multicolumn{3}{|c|}{ Type of medium } \\
\hline & Control & & $\begin{array}{l}\text { Amino acids } \\
\text { added }\end{array}$ \\
\hline \multicolumn{4}{|l|}{$\begin{array}{l}\times 10^{-6} \\
\text { mole } / L\end{array}$} \\
\hline 1.6 & $\begin{array}{l}1.59 \pm 0.13^{*} \\
\text { p }+<0.01\end{array}$ & $\mathrm{p}<0.01$ & $\begin{array}{l}4.14 \pm 0.49 \\
p<0.01\end{array}$ \\
\hline 5.0 & $\begin{array}{l}4.36 \pm 0.70 \\
\mathrm{p}<0.01\end{array}$ & $\mathrm{p}<0.02$ & $\begin{array}{l}7.86 \pm 1.89 \\
p<0.01\end{array}$ \\
\hline 50.0 & $101 \pm 15$ & $\mathrm{p}<0.01$ & $210 \pm 28$ \\
\hline
\end{tabular}

* Rates are expressed as millimicromoles of $\mathrm{Cu}$ per gram of slice per hour \pm standard error of the mean.

$\dagger$ Each $p$ value shows the level of significance of the difference between a pair of means. Each $p$ value refers to the pair of means between which it is located. was studied next. In these experiments, $\frac{1}{10} \mathrm{vol}$ of dialyzed rat serum was added to all media. Three concentrations of copper were tested, $1.6 \times$ $10^{-6}$ mole per L, $5.0 \times 10^{-6}$ mole per $\mathrm{L}$, and $50 \times 10^{-6}$ mole per L. With $3.0 \mathrm{~g}$ per $100 \mathrm{ml}$ as the approximate mean albumin concentration in

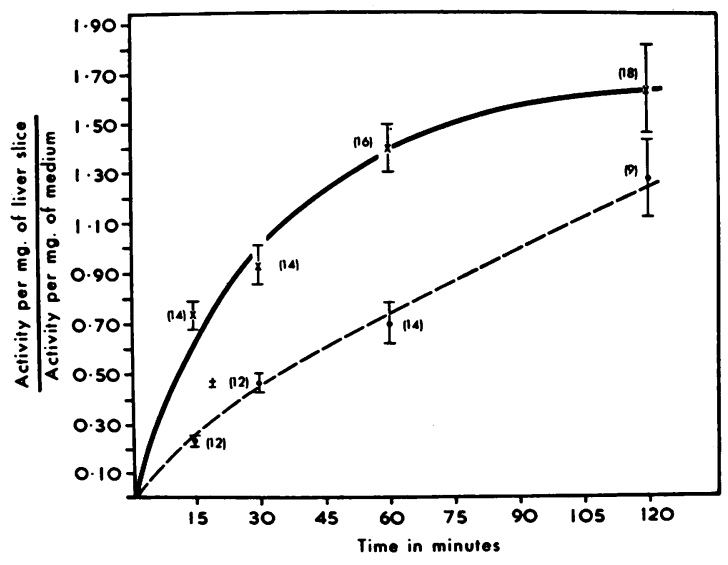

Fig. 1. Copper UPtake by LIVER SLICES IN THE PRESENCE AND ABSENCE OF ADDED AMINo ACIDS. ${ }^{84} \mathrm{Cu}$ uptake by liver slices is shown by plotting the ratio between activity per milligram of liver slice and activity per milligram of medium against time of incubation. Initial concentration of copper in the medium was $1.6 \times 10^{-8}$ mole per L. The solid circles connected by the dotted line show the mean uptakes without addition of amino acids to the medium. The crosses connected by the solid line show the mean uptakes in the presence of $20 \mathrm{~L}$-amino acids in the medium. The bars drawn across the vertical lines show the ranges of the standard errors of the means. The numbers in parentheses indicate the number of individual observations for each mean. 
rat serum (15), the $\mathrm{Cu}$ /albumin molar ratios in the media were $0.036,0.12$, and 1.2 . The experiments were carried out both in the presence and absence of added amino acids.

The results are shown in Tables II and III. In Table II, the mean ratios between the activity in the slices and the initial activity in the media that were observed after $15,30,60$, and 120 minutes of incubation are shown. If the results obtained in the absence (controls) and presence of amino acids are compared, it is apparent that higher ratios were observed in the presence of amino acids. Statistical calculations revealed that these differences are all significant at the 5\% level and, in most instances, at the $1 \%$ level. The single exception is in the presence of the highest concentration of copper after 2 hours of incubation, where the results were more scattered and, therefore, in spite of the appreciable difference between the two means, the statistical significance is doubtful. In the presence of the highest initial concentration of copper, the uptake against a concentration gradient was more marked, and uptake against a concentration gradient was observed without the addition of amino acids as well.

To demonstrate more clearly a relationship between copper uptake and copper concentration in the medium, we calculated the rates of uptake of copper by the slices, based on the activity taken up by the slices after 15 minutes of incubation. Knowing the specific activity of copper in the media and assuming that this had not changed appreciably during the short time of incubation, we expressed these rates in millimicromoles of copper taken up by a gram of slice per hour, as shown in Table III. It can be seen that the apparent rates of copper uptake increased markedly with increasing concentrations of copper in the media. This was the case both in the presence and absence of amino acids. In the presence of added amino acids, the rates were always higher than the control rates. The differences between the average rates of uptake were significant, with $\mathrm{p}<0.01$ in all but one instance, when $\mathrm{p}<0.02$.

In further experiments, we attempted to determine whether variation of the protein concentration in the medium had an effect on the uptake of copper by the liver slices. Purified human albumin was added to the media in final concentra- tions of 1.3 and $2.3 \mathrm{~g}$ per $100 \mathrm{ml}$. The higher of these concentrations was close to the normal level of albumin in rat plasma (15). Parallel experiments, in which $\frac{1}{10}$ vol of dialyzed rat serum was added to the medium (yielding an average albumin concentration of $0.3 \mathrm{~g}$ per $100 \mathrm{ml}$ ), were also carried out.

We added copper to the media to achieve a copper concentration of $1.6 \times 10^{-6}$ mole per L. After the completion of these experiments, however, we realized that the albumin preparation used in these studies was not free of copper, and that after extensive dialysis it still contained $15.8 \mu \mathrm{g}$ of copper per $\mathrm{g}$ of albumin. This meant that the initial total copper concentration in the media to which albumin had been added was not what it was intended to be, but higher. Knowing the copper content of the albumin preparation (15.8 $\mu \mathrm{g}$ per $\mathrm{g}$ ), and assuming equilibration between contaminating and added copper, we could calculate the copper contents of the media. These were found to be 5.0 $\times 10^{-6}$ mole per $\mathrm{L}$ in the case of $1.3 \mathrm{~g}$ per $100 \mathrm{ml}$ of added albumin and $7.3 \times 10^{-6}$ mole per $\mathrm{L}$ in the case of $2.3 \mathrm{~g}$ per $100 \mathrm{ml}$. Since, in the experiments outlined previously, it was shown that the concentration of copper in the medium had a definite effect on the rate of uptake of copper by the liver slices, it was evident that the results of experiments in the presence of diluted rat serum (approximately $0.3 \mathrm{~g}$ per $100 \mathrm{ml}$ albumin) and 1.3 and $2.3 \mathrm{~g}$ per $100 \mathrm{ml}$ of human albumin could not be compared without considering the respective copper concentrations of these media.

The available data could still be used, however, by choosing appropriate controls. In previous experiments we measured copper uptake into slices from media containing $\frac{1}{10}$ vol dialyzed rat serum and $5.0 \times 10^{-6} \mathrm{M}$ copper concentration; we used data from the latter experiments as controls for the present experiments with $1.3 \mathrm{~g}$ per $100 \mathrm{ml}$ albumin in the medium and $5.0 \times 10^{-6} \mathrm{M}$ concentration of copper. Controls of this nature were not available for the experiments with $2.3 \mathrm{~g}$ per $100 \mathrm{ml}$ of albumin and $7.3 \times 10^{-6} \mathrm{M}$ concentration of copper. To serve as controls, we calculated the expected uptake from media containing $\frac{1}{10} \mathrm{vol}$ dialyzed rat serum and $7.3 \times 10^{-6} \mathrm{M}$ initial copper concentration by using the rates of copper uptake determined previously at 15,30 , and 60 
minutes with $\frac{1}{10}$ vol dialyzed rat serum and $1.6 \times 10^{-6}$ and $5.0 \times 10^{-6} \mathrm{M}$ copper concentrations (Table III). The plots of these rates against the initial concentration of copper for each time in the presence and absence of amino acids fell close to two straight lines passing through the origin. Thus, by extrapolation, rates could be obtained for a copper concentration of $7.3 \times 10^{-6}$ mole per L. From these rates, we calculated the ratios of activity between the unit weights of slices and of the media by reversing calculations we used to obtain rates shown in Table III from ratios given in Table II. These calculated ratios were used as controls for the experiments with $2.3 \mathrm{~g}$ of albumin per $100 \mathrm{ml}$ of medium.

These data are shown in Figure 2. They indicate that, within the range of protein concentrations studied, amino acids do facilitate copper uptake by slices, regardless of the protein concentration. The variation of the protein concentration

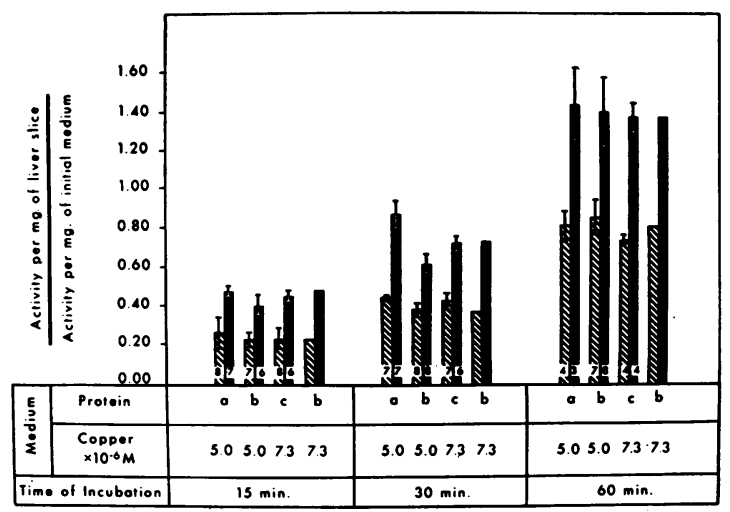

Fig. 2. EFFECT OF PROTEIN CONCENTRATION IN THE MEDIUM ON UPTAKE OF COPPER. The height of each bar represents the mean ratio between the activity per unit weight of slice and the activity per unit weight of the medium after various times of incubation, which are shown on the bottom line. The crosshatched bars indicate the uptakes without addition of amino acids to the medium; the solid bars denote uptake from media to which amino acids had been added. The numbers in the lower parts of the bars indicate the numbers of individual observations from which the means were calculated. The vertical lines at the tops of the bars indicate the ranges of the standard errors of the means. Immediately below the bars, the concentration of protein in each medium is indicated. $a=1.3 \mathrm{~g}$ per $100 \mathrm{ml}$ of human albumin, $\mathrm{b}=$ $1 / 10$ dilution of dialyzed rat serum (approximately $0.3 \mathrm{~g}$ per $100 \mathrm{ml}$ of albumin), and $\mathrm{c}=2.3 \mathrm{~g}$ per $100 \mathrm{ml}$ of human albumin. Beneath this, the initial concentration of copper in the medium is shown.

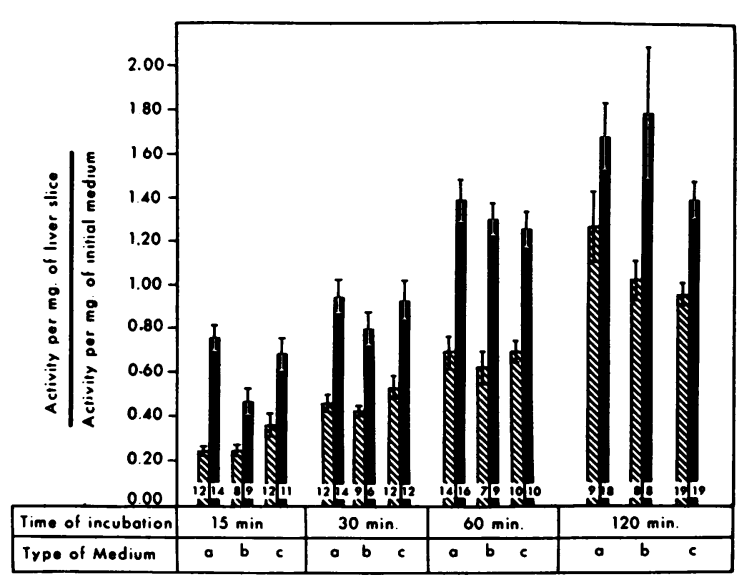

Fig. 3. EFfect of D-Glucose and sodium lactate ON COPPER UPTAKE. The height of each bar shows the mean ratio of the activity per unit weight of slice and the activity per unit weight of medium. At the bottom, the type of medium is indicated. $a=$ no glucose or sodium lactate added, $\mathrm{b}=1.1 \times 10^{-2} \mathrm{M}$ D-glucose added, and $\mathrm{c}=$ $1.1 \times 10^{-2} \mathrm{M}$ sodium lactate added. The crosshatched bars indicate uptakes (as ratios) from media to which no amino acids had been added. The solid bars show uptakes from media to which amino acids had been added. The numbers in the lower parts of the bars indicate the numbers of individual observations from which the means were calculated. Immediately beneath the bars, the intervals of incubation are shown. The vertical lines at the tops of the bars indicate the ranges of the standard errors of the means.

in the medium did not seem to influence the uptake of copper by the slices either in the presence or absence of added amino acids. The only exception to this general trend was the significant difference $(p<0.01)$ between the mean ratios observed with 0.3 and $1.3 \mathrm{~g}$ of albumin per $100 \mathrm{ml}$ after 30 minutes of incubation with added amino acids and $5.0 \times 10^{-6} \mathrm{M}$ concentration of copper. However, under the same conditions, a significant difference was not found in the absence of amino acids.

It seemed of interest to explore the possibility that the addition of substrates such as D-glucose or sodium lactate might influence the uptake of copper by the slices. Sodium lactate or D-glucose was added to the media in a final concentration of $1.1 \times 10^{-2}$ mole per L. Incubations were carried out with $1 / 10 \mathrm{vol} / \mathrm{vol}$ of dialyzed rat serum and 1.6 $\times 10^{-6} \mathrm{M}$ concentration of copper, with and without added amino acids, and in the presence and absence of the substrates. 


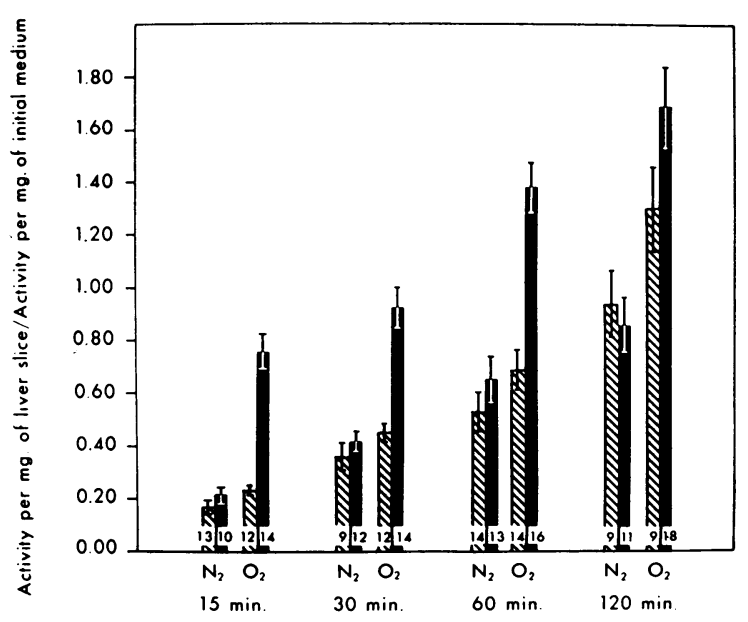

Fig. 4. EFFect of OXYGen on uptake of Copper. The height of a bar indicates the mean ratio of activity per unit weight of slice to activity per unit weight of medium. The crosshatched bars show the uptakes, as ratios, of ${ }^{64}$ copper from media without added amino acids. The solid bars show uptake by liver slices from media to which 20 L-amino acids had been added. The numbers of individual observations from which the means were calculated are shown in the lower parts of the bars. The gas that constituted $95 \%$ of the atmosphere in which the liver slices were incubated is indicated below the bars. The intervals of incubation are shown at the bottom of the Figure. The vertical lines at the tops of the bars denote the ranges of the standard errors of the means.

The results are shown in Figure 3. It can be seen that, regardless of the presence or absence of glucose or sodium lactate, amino acids do exhibit their previously observed effect on copper uptake. On the other hand, the uptake was not generally influenced by the addition of glucose or sodium lactate in the concentration used in these experiments. The minor differences that were found only in the presence of amino acids were not consistent and not significant $(p>0.05)$, except for the difference between the mean ratios in the presence and absence of glucose at $15 \mathrm{~min}$ utes $(p<0.01)$.

It seemed of interest to test whether the presence of oxygen influenced copper transport in this system. Therefore, experiments of identical design to those described first were carried out, but under an atmosphere of $95 \%$ nitrogen and 5\% carbon dioxide. The buffer for the media was gassed with $95 \%$ nitrogen and $5 \%$ carbon dioxide, and the beakers that contained the media were kept under the same atmosphere before the slices were added. Control experiments with $95 \%$ oxygen and $5 \%$ carbon dioxide were included.

In Figure 4, it can be seen that when amino acids were not added to the media, the ratios at each time were not significantly different in the presence or absence of oxygen. After 2 hours of incubation, there was a significantly lower mean ratio in the absence of oxygen. In the presence of added amino acids, the ratios in the absence of oxygen were markedly lower at each time, from 15 to 120 minutes. The differences between the mean ratios in the presence and absence of oxygen were highly significant $(p<0.01)$ at each time. Under nitrogen-carbon dioxide atmosphere, the average copper uptakes in the presence and absence of added amino acids were not significantly different, as indicated by the ratios, whereas under oxygen-carbon dioxide atmosphere, the uptake was significantly higher in the presence of amino acids.

The effect of 2,4-dinitrophenol was studied next. The design of these experiments was the same as that described for the first experiments, except that 2,4-dinitrophenol (DNP) was added to the media in final concentrations of $10^{-4}$ and $1.8 \times 10^{-4}$ mole per L. At these concentrations, DNP is an uncoupler of oxidative phosphorylation $(16,17)$. Incubations were carried out for 30 minutes only.

The results are shown in Figure 5. When amino acids were not added, DNP in the lower concentration $\left(10^{-4} \mathrm{M}\right)$ had no effect on copper uptake in this system. At the higher concentration $\left(1.8 \times 10^{-4} \mathrm{M}\right)$, there was a somewhat higher uptake of copper; the difference between this and the mean control uptake without addition of DNP, or the average uptake in the presence of the lower concentration of DNP, is significant $(p<0.02)$.

In Figure 5, it can also be seen that DNP did not abolish the facilitation of copper uptake by amino acids. In the presence of either concentration of DNP the mean ratios are not significantly different $(p>0.05)$ from the mean ratios of controls to which amino acids were added, but not DNP.

\section{Discussion}

The purpose of the model experiments reported in this paper was to determine what effect, if any, 
the addition of amino acids to the medium would have on the in vitro transfer of copper from the medium to surviving liver tissue. The results clearly indicate that amino acids promote the transfer of labeled copper from the medium to the liver slices. The facilitation of copper transport by amino acids was evident in a wide range of copper concentrations in the medium; it was uninfluenced by a variation of protein concentration in the medium within the limits of concentrations tested.

When liver slices were incubated in media in which amino acids had not been added, the transfer of copper from its protein-bound form in the medium to the slices was still observed, but it occurred at a definitely slower rate. Direct transfer of the albumin-copper complex through the cell membrane is most unlikely to proceed at the rates of copper transport observed. Alternatively, copper from its albumin-bound form may be transferred directly or as cupric ions to binding sites on the cell membrane and thence into the cell. Finally, one may also consider that when amino acids are not added, the system is still not free of amino acids. These are continuously released from the slices during incubation (18) and could easily participate in the transfer of copper.

The participation of amino acids in the rapid transfer of copper between plasma and the cells has also been observed with other systems, i.e., human red blood cells incubated in human plasma $(19,20)$ and the isolated perfused liver of the rat (19). The latter observations support the evidence presented here.

The results reported in this study allow some conclusions concerning the type of transport mechanism involved. It would seem that simple diffusion of cupric ions cannot be considered as the mode of transport, since in this transport mechanism, reaction between the transported substance $\left(\mathrm{Cu}^{++}\right)$and other molecular species (amino acids) occurs (21).

The finding that oxygen is necessary for the transport of copper which is facilitated by amino acids suggests that we may be dealing with an "active transport" that requires ATP generated by oxidative phosphorylation.

Accumulation of the transported substance against a concentration gradient is another phe-

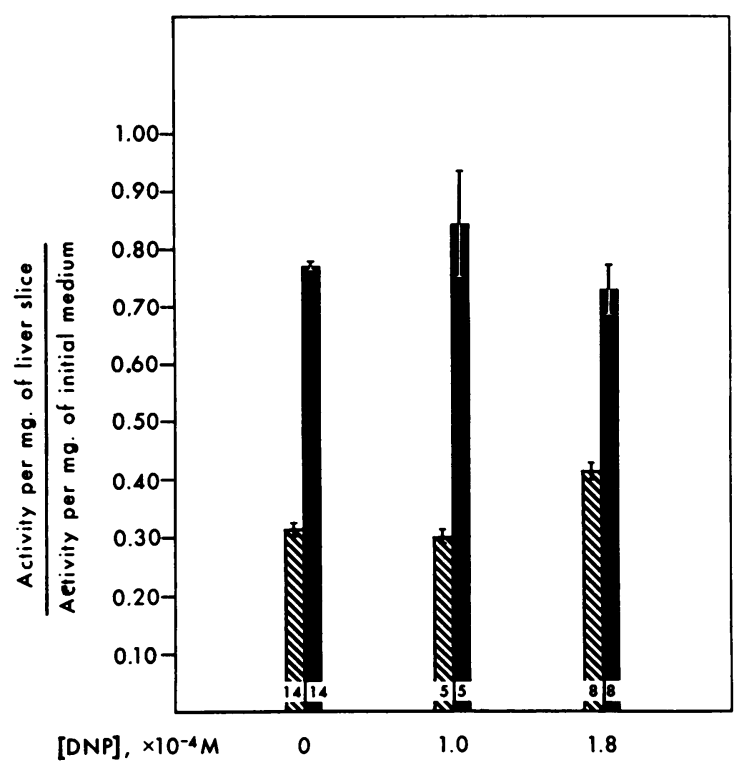

Fig. 5. EFFECT of 2,4-Dinitrophenol on UPtAKE OF COPPER. The height of each bar shows the mean ratio of the activity per unit weight of liver slice to activity per unit weight of medium. The crosshatched bars show uptakes, as ratios, from media to which no amino acids had been added. The solid bars show uptakes from media to which amino acids had been added. In the bars are shown the numbers of individual observations from which the means were calculated. Immediately below the bars are indicated the concentrations of 2,4-dinitrophenol that were added to the media. Liver slices were incubated aerobically for 30 minutes in samples of these media. The vertical lines at the tops of the bars show the ranges of the standard errors of the means.

nomenon that is characteristic of active transport (21). Accumulation of ${ }^{64} \mathrm{Cu}$ in the slices against a concentration gradient was observed in practically all experiments. This process was more apparent in the presence of added amino acids, and increased as the concentration of copper in the medium was raised. These findings, however, cannot be used as an argument in favor of the transport of copper being an active transport, since measurements of electrochemically active forms of copper were not made. In fact, the accumulation of copper against a concentration gradient in our experiments is much more likely to be due to the presence of larger numbers of strong binding sites for copper inside the cells than outside in the medium. In this situation, energy may not be required to achieve and to maintain a higher concentration inside the cell. 
The facilitation of the transport of copper by amino acids was not enhanced by the addition of substrates such as D-glucose or sodium lactate. This means that the additional energy that these substrates can provide is not required for this transport mechanism. However, the possibility that energy is required cannot be excluded on the basis of these results, since endogenous substrates could be adequate, and, secondly, the added amino acids may themselves serve as the precursors of energy-yielding substrates (22-25). Addition of glucose or sodium lactate to the incubation media without added amino acids did not increase the rates of copper uptake into the slices, and, therefore, the effect of amino acids on copper transport cannot be solely due to their being precursors of energy-yielding substrates.

Finally, it was found that DNP did not inhibit the amino acid-facilitated transport of copper. This finding suggests that energy in the form of ATP, generated by oxidative phosphorylation, is not required for the amino acid-facilitated transport of copper. Nevertheless, the possibility exists that in our system, DNP did not effectively uncouple oxidation from phosphorylation. Since this cannot be excluded, the negative findings with DNP cannot be regarded as proof against the need for energy for the amino acid-facilitated transport of copper. Further experiments are planned to decide this question.

Since the submission of our manuscript, Neumann and Silverberg have reported similar results (26). Their findings confirm and, to some extent, amplify the results presented here. Their conclusion, that the amino acid-facilitated copper transport fulfills the criteria of active transport, is not supported by adequate proof for reasons outlined by the present authors (27).

\section{Acknowledgments}

We thank Drs. Norman Aspin and S. H. Jackson for advice given during these studies, and Drs. B. Sarkar and G. R. Williams for their advice regarding the experiments with 2,4-dinitrophenol.

\section{References}

1. Neumann, P. Z., and A. Sass-Kortsak. The state of copper in human serum: evidence for an amino acid-bound fraction. J. clin. Invest. 1967, 46, 646.
2. Bush, J. A., J. P. Mahoney, H. Markowitz, C. J. Gubler, G. E. Cartwright, and M. M. Wintrobe. Studies on copper metabolism. XVI. Radioactive copper studies in normal subjects and in patients with hepatolenticular degeneration. J. clin. Invest. 1955, 34, 1766.

3. Osborn, S. B., C. N. Roberts, and J. M. Walshe. Uptake of radio-copper by the liver. A study of patients with Wilson's disease and various control groups. Clin. Sci. 1963, 24, 13.

4. Cohen, P. P. Suspending media for animal tissues in Manometric Methods, 3rd ed., W. W. Umbreit, R. H. Burris, and J. F. Stauffer, Eds. Minneapolis, Burgess Publishing Co., 1959, p. 147.

5. Eden, A., and H. H. Green. Micro-determination of copper in biological material. Biochem. J. 1940, 34, 1202.

6. Table in Blood and Other Body Fluids, P. Altman and D. S. Dittmer, Eds. Washington, D. C., Biological Handbooks, Federation of American Societies for Experimental Biology, 1961, p. 373.

7. Scientific Tables, 5th ed. J. R. Geigy, Ed. London, Jesse Broad and Co., 1956, p. 317.

8. Fritze, K. The preparation of high specific activity copper 64. Radiochim. Acta 1964, 3, 166.

9. Gubler, C. J., M. E. Lahey, G. E. Cartwright, and M. M. Wintrobe. Studies on copper metabolism. IX. The transportation of copper in blood. J. clin. Invest. 1953, 32, 405.

10. Wintrobe, M. M., G. E. Cartwright, and C. J. Gubler. Studies on the function and metabolism of copper. J. Nutr. 1953, 50, 395.

11. Harris, D. M., and J. M. Salter. Report of a component(s) in blood that accelerates urea synthesis by surviving liver slices from rats when L-amino acids are the sources of urea nitrogen. Arch. Biochem. 1966, 113, 173.

12. Stadie, W. C., and B. C. Riggs. Microtome for the preparation of tissue slices for metabolic studies of surviving tissues in vitro. J. biol. Chem. 1944, 154, 687.

13. Gosset, W. S. The probable error of a mean (by "Student"). Biometrika 1908, 6, 1.

14. Fisher, R. A. On a distribution yielding the error functions of several well-known statistics. Proceedings of the International Mathematical Congress (Toronto), 1924, S. C. Fields, Ed. Toronto, University of Toronto Press, 1928, vol. 2, p. 805.

15. Table in Blood and Other Body Fluids, P. Altman and D. S. Dittmer, Eds. Washington, D. C., Biological Handbooks, Federation of American Societies for Experimental Biology, 1961, p. 77.

16. Loomis, W. F., and F. Lipmann. Reversible inhibition of the coupling between phosphorylation and oxidation. J. biol. Chem. 1948, 173, 807.

17. Frantz, I. D., Jr., P. C. Zamecnik, J. W. Reese, and M. L. Stephenson. The effect of dinitrophenol on the incorporation of alanine labeled with radio- 
active carbon into the proteins of slices of normal and malignant rat liver. J. biol. Chem. 1948, 174, 773.

18. Kritzmann, M. G. The mechanism of formation of amino acids in surviving animal tissues from pyruvate and ammonia. J. biol. Chem. 1947, 167, 77.

19. Sass-Kortsak, A., D. M. Harris, S. J. Goodman, M. Hawke, and R. H. Smuckler. The possible role of amino acids in copper transport (abstract). Canad. med. Ass. J. 1965, 92, 368.

20. Neumann, P. Z., and M. Silverberg. Copper transport in erythrocytes in normals and in Wilson's disease (abstract). Proc. Canad. Fed. biol. Soc. 1965, 8, 49.

21. Park, C. R. General aspects of transport phenomena in Membrane Transport and Metabolism, A. Kleinzeller and A. Kotyk, Eds. New York, Academic Press, 1960, p. 19.
22. Cohn, R. Zur Frage der Zuckerbildung aus Einweiss. Hoppe-Seylers Z. physiol. Chem. 1899, 28, 211.

23. Pflüger, E. Nachschrift. Pflügers Arch. ges. Physiol. 1910, 131, 302.

24. Wilson, R. H., and H. B. Lewis. Comparative studies of the metabolism of amino acids. III. The formation of glycogen after oral administration of amino acids to white rats. J. biol. Chem. 1929-30, 85, 559.

25. Krebs, H. A. Gluconeogenesis from amino acids in Mammalian Protein Metabolism, H. N. Munro and J. B. Allison, Eds. New York, Academic Press, 1964, vol. 1, p. 162.

26. Neumann, P. Z., and M. Silverberg. Active copper transport in mammalian tissues-a possible role in Wilson's disease. Nature (Lond.) 1966, 210, 414.

27. Sass-Kortsak, A., and D. I. M. Harris. Copper transport in mammalian tissues. Nature (Lond.) 1966, 211, 852. 